\title{
Cooperative Spectrum Sensing based on Majority decision under CFAR and CDR constraints
}

\author{
Giuseppe Caso*, Luca De Nardis*, Guido Carlo Ferrante*, Maria-Gabriella Di Benedetto* \\ * DIET Department \\ Sapienza University of Rome \\ Rome, Italy \\ Email: \{caso, lucadn, ferrante, dibenedetto\}@ newyork.ing.uniroma1.it
}

\begin{abstract}
This work addresses the impact of sensing decision fusion rules and operative constraints on the performance of hard decision cooperative spectrum sensing schemes for cognitive radio networks. The work focuses on cooperative spectrum sensing under two sensing operating modes: the Constant False Alarm Rate (CFAR) and the Constant Detection Rate (CDR) mode. Analytical solutions for network probability of false alarm and of detection under the assumption that all nodes share the same individual probability of false alarm or detection, respectively, are reviewed, and corresponding exact analytical expressions for such probabilities are expressed for OR and AND fusion rules. In the case of the Majority fusion rule novel analytical approximations for the required values of individual probability of false alarm in CFAR mode and individual probability of detection in CDR mode, respectively, are derived. The validity of the approximations is confirmed by means of numerical simulations, and the impact of errors in the estimation of average SNR for the signal of the primary user at the receivers of the cognitive network is analyzed by network simulations.
\end{abstract}

\section{INTRODUCTION}

Advancements in wireless technology make the RF spectrum a key resource, and consequently, its efficient use is of paramount importance. Dynamic Spectrum Access using Cognitive Radio (CR) ([1][2]) is widely considered a feasible solution for this issue [3]. Until recently, research about CR mainly focused on Spectrum Sensing Cognitive Radio, that considers the spectrum as the source of stimuli and information to be processed in a cognitive way [4]. By adopting Spectrum Sensing (SS), a single cognitive transceiver aims at identifying the currently unused spectrum by detecting the presence/absence of other users on the channel. As a result of sensing, each user in a Cognitive Radio Network (CRN) creates a map of the channel state based on local observations, leading to variable reliability of the sensing decisions in both space and time. In order to cope with this limitation, Cooperative Spectrum Sensing (CSS) was proposed: the whole set of nodes in the CRN, or a selected subset of them, shares the results of sensing phase to other nodes directly or to a central unit [5]. Increased probability of correct identification of spectrum usage can be, therefore, obtained thanks to cooperation between CR terminals, which exchange information in order to obtain a common map of the channel status. Although regulators in US, Europe and UK introduced geolocation databases as a solution to determine the presence of Primary Users (PUs), FCC in US left open the possibility of relying on sensing, provided that it guarantees the required performance. In this context, CSS becomes very important when detection of the PU is transmitter-based and the PU receiver is located in the Secondary Users (SUs) transmission range (Hidden PU problem). Performance of SS is typically measured by the following indicators: Probability of Detection $\left(P_{\mathrm{d}}\right)$, complementary Probability of Miss-Detection $\left(P_{\mathrm{md}}\right)$ and Probability of False Alarm $\left(P_{\mathrm{fa}}\right)$. Note the existing tradeoff between $P_{\mathrm{md}}$ and $P_{\mathrm{fa}}$ : high $P_{\mathrm{md}}$ implies that the presence of the PU is not detected by the SU with high probability, increasing interference to the PU. Conversely, high $P_{\mathrm{fa}}$ implies that the SU decides for the PU presence with high probability also when the PU is not transmitting, decreasing the SU spectrum utilization. From the PU standpoint, $P_{\mathrm{d}}$ of $\mathrm{SU}$, or, in the cooperative scenario, of whole $\mathrm{CRN}$, should be maximized in order to minimize interference, while from the $\mathrm{SU}(\mathrm{CRN})$ standpoint, $P_{\mathrm{fa}}$ should be minimized in order to increase spectrum utilization when PU is silent. The two different perspectives lead to the definition of two different SS operating modes: the Constant False Alarm Rate (CFAR) and the Constant Detection Rate (CDR) mode, where it is assumed that the probability of false alarm or the probability of detection, respectively, are constant and equal to given target values. This work focuses on CFAR and CDR sensing modes in a centralized CSS network. Three different hard decision fusion rules, OR, Majority and AND rules, are analyzed. Moving from the observation that OR/AND rules can be seen as special cases of the Majority one and that many previous works on hard CSS, either focus in particular on OR rule analysis (that is, with particular assumptions, the rule with the most notable cooperative gain), or analyse the rules without providing a closed formula for the evaluation of the performance, this work fills the gap in analytical modeling of CSS by introducing, in particular for the Majority rule, a novel analytical approximation to derive the requirement on $P_{\mathrm{fa}}$ and $P_{\mathrm{d}}$ for a single node in CFAR and CDR modes. The analysis is then complemented by a comparative analysis via computer simulation.

The paper is organized as follows: Section II introduces the theoretical setting for CSS and the system model used for later analyses; Sections III and IV focus, respectively, on CFAR and CDR sensing modes, providing expressions for performance indicators for three different hard fusion rules at 
the FC; Section V presents and discusses results of extensive performance evaluation via simulation of the previous operating modes, under different hypotheses and setup. Finally, Section VI draws conclusions.

\section{System Model for COOPERATIVE SPECTRUM SENSING}

Spectrum Sensing is a very important tool for a CR device, in order to measure, learn and be aware of parameters related to the radio channel; as a consequence, huge research efforts focused on the definition of SS techniques. A widely adopted and simple choice consists of using energy detection, referred to hereinafter as Energy Detector Spectrum Sensing (ED-SS), aiming at low computational and implementation complexities. In ED-SS, CR receivers do not need any knowledge on the PUs signal, measuring the energy of the received waveform over an observation time window of $T$ (seconds) and comparing the test statistic $Y$, that approximates the signal energy in the interval $(0, T)$, with a threshold $\lambda$, whose optimum value depends on the noise floor [6]: if the evaluated energy is larger (resp. lower) than the threshold, then SU decides for PU presence (resp. absence). Framing this problem into a decision problem, the two hypotheses, denoted by $\mathcal{H}_{0}$ and $\mathcal{H}_{1}$, are thus defined as follows:

$$
\begin{aligned}
& \mathcal{H}_{0}: Y<\lambda, \\
& \mathcal{H}_{1}: Y \geq \lambda .
\end{aligned}
$$

In a Local Spectrum Sensing (LSS) scenario, a CR node monitors the licensed frequency band and opportunistically transmits when it does not detect presence of any PUs, where decision about PUs presence on the channel is not related to SS results of other SUs in the surrounding environment.

In a non-fading environment, denoting with $\gamma$ the PU signalto-noise ratio (SNR) at the $\mathrm{SU}$ receivers within a bandwidth $W$ (hertz) and assuming for the test statistic $Y$, in hypothesis $\mathcal{H}_{0}$ and $\mathcal{H}_{1}$, respectively, central and non-central (with noncentrality parameter of $2 \gamma$ ) chi-square distributions with $2 T W$ degrees of freedom, $P_{\mathrm{d}}$ and $P_{\mathrm{fa}}$ are as follows:

$$
\begin{aligned}
& P_{\mathrm{d}}=P\left\{Y>\lambda \mid \mathcal{H}_{1}\right\}=Q_{m}(\sqrt{2 \gamma}, \sqrt{\lambda}), \\
& P_{\mathrm{fa}}=P\left\{Y>\lambda \mid \mathcal{H}_{0}\right\}=\frac{\Gamma\left(m, \frac{\lambda}{2}\right)}{\Gamma(m)} .
\end{aligned}
$$

where it is assumed, for notation simplicity, that TimeBandwidth product $T W$ is the integer number $m, \Gamma(\cdot)$ and $\Gamma(\cdot, \cdot)$ are the complete and incomplete gamma functions, and $Q_{m}(\cdot, \cdot)$ is the generalized Marcum $Q$-function, defined from the $I_{m-1}(\cdot)$ modified Bessel function of $(m-1)$ th order [5].

It is useful to have means for rapidly computing false alarm and detection probabilities for given $m$ and $\gamma$. For large values of $m$, well describing the physical model of the current work, the Gaussian Approximation can be applied to the test statistic $Y$ under either $\mathcal{H}_{0}$ or $\mathcal{H}_{1}$. Following [6] again, under the $\mathcal{H}_{0}, Y$ is the sum of $2 m$ statistically independent random variables. Therefore, since $\mathbb{E}[Y]=2 m$ and $\operatorname{Var}[Y]=4 m$,
$Y$ is distributed as a Gaussian random variable denoted by $\mathcal{N}(2 m, 4 m)$, and the $P_{\mathrm{fa}}$ is given by:

$$
P_{\mathrm{fa}}=\frac{1}{\sqrt{8 \pi m}} \int_{Y}^{\infty} e^{-\frac{(x-2 m)^{2}}{8 m}} d x=\frac{1}{2} \operatorname{erfc}\left(\frac{\lambda-2 m}{2 \sqrt{2 m}}\right) .
$$

Under $\mathcal{H}_{1}, \mathbb{E}[Y]=2 m+2 \gamma$ and $\operatorname{Var}[Y]=4(m+2 \gamma)$, and therefore $Y \sim \mathcal{N}(2 m+2 \gamma, 4(m+2 \gamma))$. $P_{\mathrm{d}}$ is given by:

$$
P_{\mathrm{d}}=\frac{1}{2} \operatorname{erfc}\left(\frac{\lambda-2 m-2 \gamma}{2 \sqrt{2} \sqrt{m+2 \gamma}}\right) \text {. }
$$

When the channel gain $h$ is varying due to shadowing/fading, (1) gives probability of detection conditioned on the instantaneous $\gamma$. In this case, $P_{\mathrm{d}}$ is derived by averaging (1) over fading statistics, that is:

$$
P_{\mathrm{d}}=\int_{\gamma} Q_{m}(\sqrt{2 \gamma}, \sqrt{\lambda}) f_{\Gamma}(\gamma) d \gamma,
$$

where $f_{\Gamma}(\gamma)$ is the pdf of SNR under fading.

In order to improve LSS performance, several authors have proposed collaboration among SUs, defining the so called Cooperative Spectrum Sensing, relying on either distributed and centralized organization [5]. In hard decision distributed CSS schemes, each CR node takes and shares an independent decision on the PU presence. Consequently, each CR node receives locally the detection decision from its neighbors SUs and applies a fusion rule to obtain its final detection decision. The hard fusion rule can be the $k$ out of $n$ rule: if $k$ or more nodes decide the hypotheses $\mathcal{H}_{1}$, then the node will decide for $\mathcal{H}_{1}$. When $k=1$, the rule becomes the OR rule; when $k=n$ the fusion rule works as the AND rule; when $k=(n+1) / 2$, the fusion rule becomes the Majority rule.

Let $N$ denote the number of collaborating SUs, experiencing independent and identically distributed fading/shadowing with same average SNR. It was shown that when sensors are conditionally independent (as in this case), optimal decision rule for individual sensors is Likelihood Ratio Test (LRT) [7]. However, optimum individual thresholds are not necessarily equal and it is generally hard to derive them. Let us assume that all SUs employ ED rather than LRT and use the same decision rule (with threshold $\lambda$ ). If a SU receives decisions from $N-1$ other users and it applies the generic $n$-out-of$N$, then the probabilities of detection and false-alarm for the collaborative scheme ( $Q_{\mathrm{d}}$ and $Q_{\mathrm{fa}}$, respectively) are:

$$
\begin{aligned}
Q_{\mathrm{d}} & =\sum_{k=n}^{N}\left(\begin{array}{c}
N \\
k
\end{array}\right) P_{\mathrm{d}}^{k}\left(1-P_{\mathrm{d}}\right)^{N-k}, \\
Q_{\mathrm{fa}} & =\sum_{k=n}^{N}\left(\begin{array}{c}
N \\
k
\end{array}\right) P_{\mathrm{fa}}^{k}\left(1-P_{\mathrm{fa}}\right)^{N-k},
\end{aligned}
$$

where $P_{\mathrm{d}}$ and $P_{\mathrm{fa}}$ are the individual probabilities of detection and false alarm as defined before. Using the OR rule (1 out of $N$ ), (6) (7) become:

$$
\begin{gathered}
Q_{\mathrm{d}}=1-\left(1-P_{\mathrm{d}}\right)^{N}, \\
Q_{\mathrm{fa}}=1-\left(1-P_{\mathrm{fa}}\right)^{N} .
\end{gathered}
$$


Formulas for Majority rule $(\lceil N / 2\rceil$ out of $N)$ are:

$$
\begin{aligned}
Q_{\mathrm{d}} & =\sum_{k=\lceil N / 2\rceil}^{N}\left(\begin{array}{l}
N \\
k
\end{array}\right) P_{\mathrm{d}}^{k}\left(1-P_{\mathrm{d}}\right)^{N-k}, \\
Q_{\mathrm{fa}} & =\sum_{k=\lceil N / 2\rceil}^{N}\left(\begin{array}{l}
N \\
k
\end{array}\right) P_{\mathrm{fa}}^{k}\left(1-P_{\mathrm{fa}}\right)^{N-k} .
\end{aligned}
$$

Lastly, for the AND ( $N$ out of $N$ ), one obtains:

$$
\begin{aligned}
Q_{\mathrm{d}} & =P_{\mathrm{d}}^{N}, \\
Q_{\mathrm{fa}} & =P_{\mathrm{fa}}^{N} .
\end{aligned}
$$

When the network is infrastructure based, there will be a Base Station (BS), also known as Fusion Center (FC), for CSS management. The BS will be the only device in the network that, using the LSS decisions by the SUs, will apply a fusion rule and then will broadcast the cooperative decision. After receiving the authorization from the $\mathrm{BS}$, all the cognitive users initiate the spectrum sensing independently and then forward their observations to it. If the channels between SUs and FC are perfect, $Q_{\mathrm{fa}}, Q_{\mathrm{d}}$ and $Q_{\mathrm{md}}$ are defined as before.

\section{Operating Mode 1: Constant False Alarm Rate (CFAR)}

In this operating mode it is assumed that the overall CRN has fixed a target probability of false alarm $\bar{Q}_{\mathrm{fa}}$, in order to optimize as better as possible the usage of spectrum opportunities when the licensed channel is free. Given $\bar{Q}_{\mathrm{fa}}$, the corresponding $\bar{P}_{\mathrm{fa}}$ can be obtained using the chosen fusion rule formula. This leads to the evaluation of the threshold $\lambda$, inverting (3), and the consequent evaluations of $P_{\mathrm{d}}$ and $Q_{\mathrm{d}}$, for a given value of $\gamma$. In this case, the generic formulation of the threshold $\lambda$ is as follows:

$$
\lambda^{\mathrm{CFAR}}=\operatorname{erfc}^{-1}\left(2 \bar{P}_{\mathrm{fa}}\right)[2 \sqrt{2 m}]+2 m .
$$

After showing the procedure for AND and OR rules, in the following subsection a numerical approximation of (11) for Majority rule is proposed, allowing to obtain a good estimate of $\bar{P}_{\mathrm{fa}}$ without inverting (11).

\section{A. Hard Decision Fusion Rules}

OR/AND rules - Inverting respectively (9) and (13), one obtains:

$$
\begin{array}{ll}
\bar{P}_{\mathrm{fa}}=1-\sqrt[N]{1-\bar{Q}_{\mathrm{fa}}} & \text { OR rule, } \\
\bar{P}_{\mathrm{fa}}=\sqrt[N]{\bar{Q}_{\mathrm{fa}}} & \text { AND rule. }
\end{array}
$$

At this point, it is possible to compute $\lambda^{\mathrm{CFAR}}$ using one of the previous formulas for $\bar{P}_{\mathrm{fa}}$, in order to subsequently obtain $P_{\mathrm{d}}$ and $Q_{\mathrm{d}}$.
Majority rule - Instead of inverting (11), we propose to approximate it. First of all, we apply the de Moivre-Laplace theorem to the argument of (11):

$$
\left(\begin{array}{c}
N \\
k
\end{array}\right) \bar{P}_{\mathrm{fa}}^{k}\left(1-\bar{P}_{\mathrm{fa}}\right)^{N-k} \approx \frac{1}{\sqrt{2 \pi N \bar{P}_{\mathrm{fa}}\left(1-\bar{P}_{\mathrm{fa}}\right)}} e^{\frac{-\left(k-N \bar{P}_{\mathrm{fa}}\right)^{2}}{2 N \bar{P}_{\mathrm{fa}}\left(1-\bar{P}_{\mathrm{fa}}\right)}}
$$

Using (17) in (11), one has

$$
\bar{Q}_{\mathrm{fa}} \approx \sum_{k=\lceil N / 2\rceil}^{N} \frac{1}{\sqrt{2 \pi N \bar{P}_{\mathrm{fa}}\left(1-\bar{P}_{\mathrm{fa}}\right)}} e^{\frac{-\left(k-N \overline{\mathrm{f}}_{\mathrm{f}}\right)^{2}}{2 N \mathrm{P}_{\mathrm{fa}}\left(1-P_{\mathrm{fa}}\right)}} .
$$

For $N \rightarrow \infty$, the summation, unless of a multiplicative factor, converges to the Riemann integral of the argument. We indeed introduce then the integral but, in our approximation, the upper limit is $\infty$, introducing thus an additional term to the summation: this term, indeed, is an $\operatorname{erfc}($.$) , converging to$ zero when $N \rightarrow \infty$. For this reason one can expect that, given a finite value of $N$, the summation is well matched with the integral and that the additional term is negligible. Indeed, one obtains:

$\left|\sum_{k=\lceil N / 2\rceil}^{N} \frac{1}{\sqrt{2 \pi N \bar{P}_{\mathrm{fa}}\left(1-\bar{P}_{\mathrm{fa}}\right)}} e^{\frac{-\left(k-N \bar{P}_{\mathrm{fa}}\right)^{2}}{2 N P_{\mathrm{fa}}\left(1-P_{\mathrm{fa}}\right)}}-\int_{a}^{\infty} \frac{1}{\sqrt{\pi}} e^{-x^{2}} d x\right| \leqslant \delta(N)$

where $a=\frac{\lceil N / 2\rceil-\frac{1}{2}-N \bar{P}_{\mathrm{fa}}}{\sqrt{2 N \overline{\mathrm{P}}_{\mathrm{fa}}\left(1-\bar{P}_{\mathrm{fa}}\right)}}$ and $\delta(N)$ is a positive number decreasing with N. Finally, one can write:

$$
\left|\sum_{k=\lceil N / 2\rceil}^{N}\left(\begin{array}{c}
N \\
k
\end{array}\right) \bar{P}_{\mathrm{fa}}^{k}\left(1-\bar{P}_{\mathrm{fa}}\right)^{N-k}-\frac{1}{2} \operatorname{erfc}(a)\right| \leqslant \varepsilon(N)
$$

where $\varepsilon(N)$ is, again, a positive number decreasing with $\mathrm{N}$, representing the discrepancy between the theoretical and the proposed approximation; finally, we note that, respect to the Riemann integral definition, the lower limit $a$ is shifted of a value $-\frac{1}{2}$ that, as heuristically found, leads to a very accurate approximation. $Q_{\mathrm{fa}}$ can be thus approximated by using $\operatorname{erfc}(a)$ function.

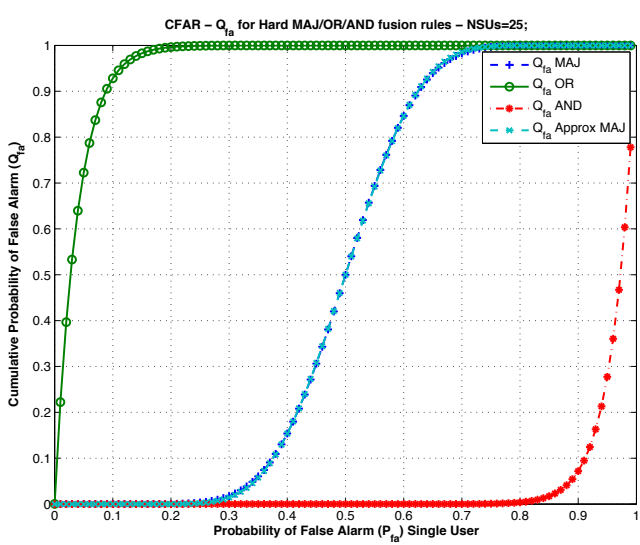

Fig. 1. CFAR - Target $\bar{Q}_{\mathrm{fa}}$ vs Target $\bar{P}_{\mathrm{fa}}$

Figure 1 compares the $\bar{Q}_{\mathrm{fa}}$ obtained with OR, AND, exact Majority formula, and the proposed approximation, and shows 
the good match between exact and approximated Majority formulas, confirming the validity of the approximation, that can be used to easily get the $\bar{P}_{\mathrm{fa}}$ required to obtain a target $\bar{Q}_{\mathrm{fa}}$. Figure 2 presents the value of $\varepsilon$ as a function of the number of SUs, and shows that the approximated formula gets more and more accurate as the number of SUs increases.

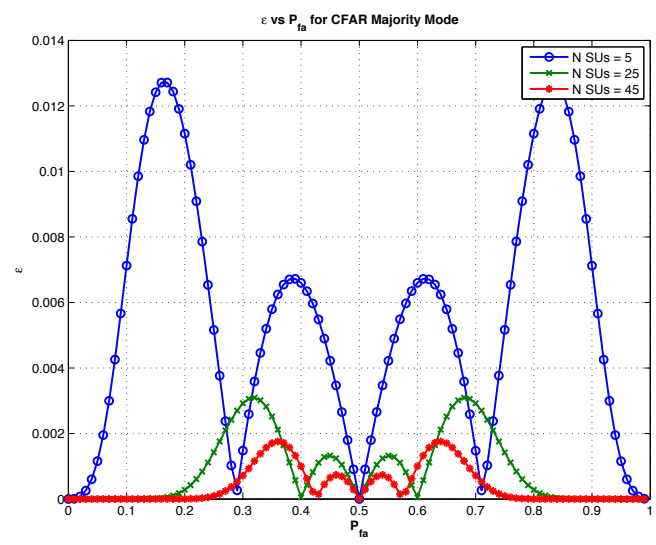

Fig. 2. Theoretical vs Approximated formula for $\bar{Q}_{\mathrm{fa}}$ in terms of $\varepsilon(N)$ for CFAR Majority Operating Mode

\section{Operating Mode 2: Constant Detection Rate (CDR)}

In CDR mode it is assumed that a target $\bar{Q}_{\mathrm{d}}$ was selected for the CRN, determine so to meet the coexistence constraints related to licensed PU. Given $\bar{Q}_{\mathrm{d}}$, the corresponding $\bar{P}_{\mathrm{d}}$, is obtained by inverting the formula of the chosen fusion rule. This leads to the evaluation of the threshold $\lambda$, applying (4) for a given value of $\gamma$, and the consequent evaluations of $P_{\mathrm{fa}}$ and $Q_{\mathrm{fa}}$. In this case, the generic formulation of $\lambda$ is as follows

$$
\lambda^{\mathrm{CDR}}=\operatorname{erfc}^{-1}\left(2 \bar{P}_{\mathrm{d}}\right)[2 \sqrt{2(m+2 \gamma)}]+2(m+\gamma) .
$$

\section{A. Hard Decision Fusion Rules}

OR/AND rules - Inverting respectively (8) and (12), one obtains:

$$
\begin{array}{ll}
\bar{P}_{\mathrm{d}}=1-\sqrt[N]{1-\bar{Q}_{\mathrm{d}}} & \text { OR rule } \\
\bar{P}_{\mathrm{d}}=\sqrt[N]{\bar{Q}_{\mathrm{d}}} & \text { AND rule. }
\end{array}
$$

At this point, it is possible to compute $\lambda^{\mathrm{CDR}}$ using one of the previous formulas for $\bar{P}_{\mathrm{d}}$, in order to subsequently obtain $P_{\mathrm{fa}}$ and $Q_{\mathrm{fa}}$.

Majority rule - In this case an approximation for (10) is proposed. Following the same steps as in Section III , the following inequality is obtained:

$$
\left|\sum_{k=\lceil N / 2\rceil}^{N}\left(\begin{array}{l}
N \\
k
\end{array}\right) \bar{P}_{\mathrm{d}}^{k}\left(1-\bar{P}_{\mathrm{d}}\right)^{N-k}-\frac{1}{2} \operatorname{erfc}(a)\right| \leqslant \varepsilon(N) .
$$

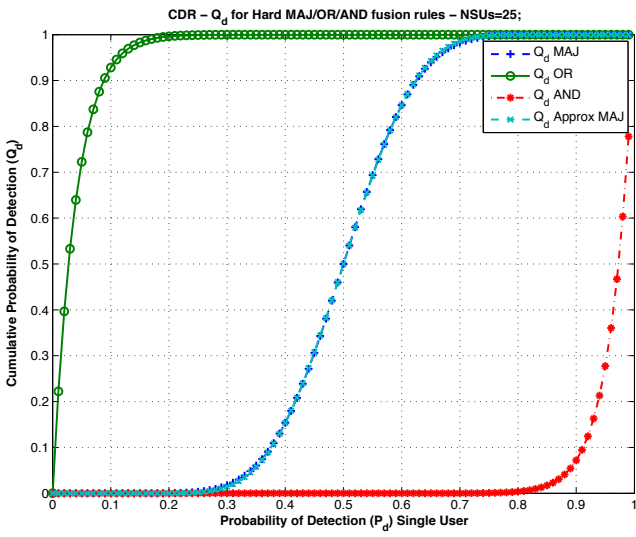

Fig. 3. CDR - Target $\bar{Q}_{\mathrm{d}}$ vs Target $\bar{P}_{\mathrm{d}}$

Figure 3 shows the match between approximated and exact formulas for $\bar{Q}_{\mathrm{d}}$ as a function of $\bar{P}_{\mathrm{d}}$. The role of the number of SUs on the accuracy of the approximation is comparable to the CFAR case discussed in Figure 2.

\section{RESUlTS AND DisCUSSIONS}

The theoretical derivations presented in previous sections were compared with simulation results in a scenario characterized by varying average SNR for primary signal at secondary receivers.

\section{A. Simulation Environment and Parameters}

The performance evaluation was carried out within the OMNeT++ simulation environment, taking advantage of the MiXiM framework [8]. In the considered simulation scenario a PU is located in the top left corner of a square area of $20 \times 20 \mathrm{~km}^{2}$; it uses a fixed transmitter power $(200 \mathrm{~kW})$ and a single DVB-T $8 \mathrm{MHz}$ channel in the UHF bandwidth. A CRN formed by $25 \mathrm{SUs}$ is located at the lower right part of the playground, within a $700 \times 700 \mathrm{~m}^{2}$ area, centered on the position of the FC. The SUs communicate among them with a maximum transmission power of $110 \mathrm{~mW}$. A $20 \mathrm{MHz} 802.11$ CRN control channel used to exchange generic control packets and sensing information is also simulated. Each run covers 1 hour of simulated time, during which each SU takes a local decision within a sensing phase of $50 \mu$ s and then transmits it to the FC during the subsequent exchange phase of 1 second. The FC takes a global decision every 5 seconds.

\section{B. Simulation Results}

Figures 4 and 5 show achievable $Q_{\mathrm{d}}$ (CFAR mode) and $Q_{\mathrm{fa}}(\mathrm{CDR}$ mode), respectively as a function of the requested targets and for different values of $\gamma$. Figures focus on Majority rule due to space constraints. Perfect knowledge about the number of collaborating SUs $(N=25)$ and the average SNR $(\gamma=[0: 5: 30] \mathrm{dB})$, is assumed, leading in turn to optimal choice of ED threshold $\lambda$. As a result, all target $\bar{Q}_{\mathrm{fa}}$ in CFAR mode and all target $\bar{Q}_{\mathrm{d}}$ in CDR mode are met. As expected, for low $\gamma$ values, the CRN should not set 
too stringent targets as this actually leads to worse global cooperative performance: figures show that a low $\bar{Q}_{\mathrm{fa}}$ leads to poor detection performance for CFAR, while high $\bar{Q}_{\mathrm{d}}$ lead to unacceptable false alarm rates for CDR. For high $\gamma$ values the performance greatly increase in both operating modes.

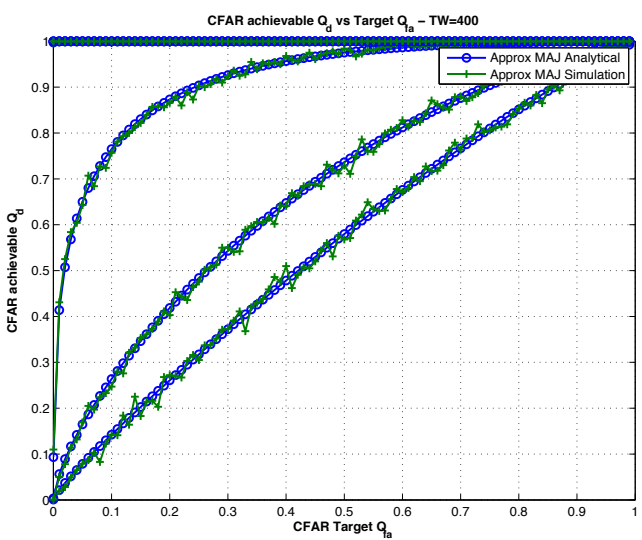

Fig. 4. CFAR - Achievable $Q_{\mathrm{d}}$ vs Target $\bar{Q}_{\mathrm{fa}}$

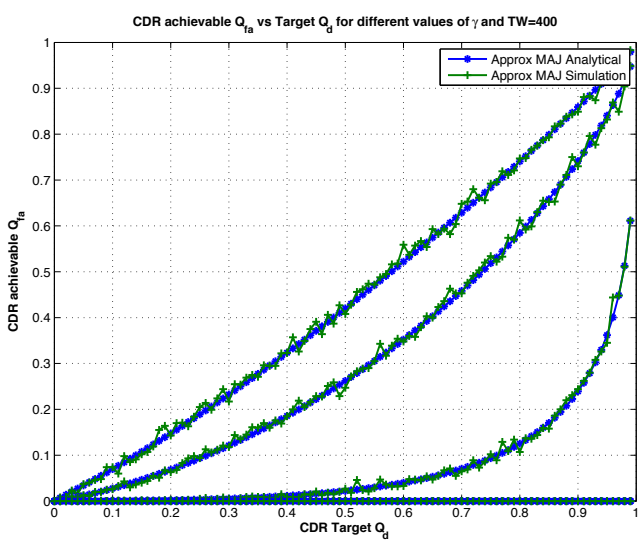

Fig. 5. $\quad$ CDR - Achievable $Q_{\mathrm{fa}}$ vs Target $\bar{Q}_{\mathrm{d}}$

As mentioned above, the number of involved SUs and the expected average PU-related SNR $\gamma$ sensed by the CRN are required to define the threshold $\lambda$. As regards $N$, a reasonably good estimate can be obtained by relying on a training phase involving SUs and FC. Estimating $\gamma$ is however much harder, at least under the assumption of no a-priori knowledge about the PU. Focusing on CDR mode, Figure 6 shows the impact of errors in $\gamma$ estimate on the target $\bar{Q}_{\mathrm{d}}$ achievability, assuming $\bar{Q}_{\mathrm{d}}=0.9, N=25$ and $\bar{\gamma}=5 \mathrm{~dB}$. As expected, for values of $\gamma$ lower than $\bar{\gamma}$, the CRN is not able to meet the target $\bar{Q}_{\mathrm{d}}$. The floor observed for the lowest value of $\gamma$ is due to the hypothesis of continuous activity of the PU (that allows the evaluation of $Q_{\mathrm{d}}$ even if in a very low- $\gamma$ case). Moreover, evaluating the three thresholds of the fusion rules, the verified inequality is $\lambda_{\mathrm{MAJ}}>\lambda_{\mathrm{OR}}>\lambda_{\mathrm{AND}}$. This explains why the Majority rule behaves as a lower (upper) bound when the $\lambda$ estimate is too optimistic (pessimistic).

\section{CONCLUSIONS}

This work analyzed CSS schemes under CFAR and CDR operative modes, adopting OR, AND and Majority decision rules. Novel approximations for the network probability of false alarm (CFAR) and detection (CDR) as a function of individual probabilities were proposed, that allow to derive individual performance requirements. The approximations were validated by means of simulations, and the role of errors in the estimation of parameters used for setting individual performance requirements was investigated; results suggest that effective operation of a CSS scheme requires accurate information on the operative environment.

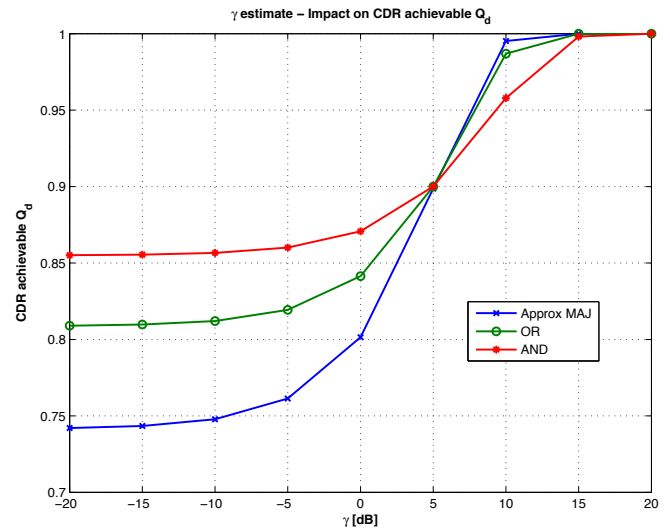

Fig. 6. CDR achievable $Q_{\mathrm{d}}$ - ED-SS threshold $\lambda$ is fixed in order to achieve $\bar{Q}_{\mathrm{d}}=0.9$ if $\bar{\gamma}=5 \mathrm{~dB}$

\section{ACKNOWLEDGMENT}

This paper has been supported by the ICT-ACROPOLIS Network of Excellence, FP7 project n. 257626 and by COST Action IC0902 "Cognitive Radio and Networking for Cooperative Coexistence of Heterogeneous Wireless Networks".

\section{REFERENCES}

[1] Mitola, J.; The Software Radio - IEEE National Telesystems Conference, 1992.

[2] Mitola, J.; Maguire G. Q.; Cognitive radio: Making Software Radios more personal - IEEE Personal Communications, Aug. 1999.

[3] FCC - Notice of proposed rule making and order: In the Matter of Facilitating Opportunities for Flexible, Efficient, and Reliable Spectrum Use Employing Cognitive Radio Technologies - ET Docket n. 03-108, Dec. 2003

[4] Axell, E.; Leus, G; Larsson, E.G.; Poor, H.V.; Spectrum Sensing for Cognitive Radio: State-of-the-Art and Recent Advances - in Signal Processing Magazine, IEEE, Volume: 29, Issue: 3, Pages: 101-116, May 2012

[5] Ghasemi, A.; Sousa, E. S.; Collaborative Spectrum Sensing for Opportunistic Access in Fading Environments - New Frontiers in Dynamic Spectrum Access Networks, 2005. DySPAN 2005. 2005 First IEEE International Symposium, pp.131-136, Baltimore, MD, USA, Nov. 2005.

[6] Urkowitz, H.; Energy Detection of unknown deterministic signals - Proc. IEEE, vol.LV pp. 523-531, Apr. 1967.

[7] Varshney, P.K.; Distributed Detection and Data Fusion New York: Springer-Verlag, 1997.

[8] Koepke, A.; Swigulski, M.; Wessel, K.; Willkomm, D.; Klein Haneveld, P.T.; Parker, T.E.V.; Visser, O.W.; Lichte, H.S.; Valentin, S.; Simulating Wireless and Mobile Networks in OMNeT++: The MiXiM Vision. 